\title{
Un ejemplo de formación científica y humanista: Darwin y el darwinismo en La Edad de Oro
}

"An example of scientific and humanist formation:
Darwin and the darwinism in The Age of Cold

\begin{tabular}{l}
\hline Luis Ernesto Martínez González \\
\hline Universidad de Matanzas \\
\hline
\end{tabular}

\section{Resumen}

Esta ponencia aborda un tema vinculado a la presencia de la ciencia como parte de la formación humanística con que José Martí pretendió educar a los niños de América mediante su revista La Edad de Oro. En este sentido, se asume una temática que está implícita en varios de sus textos, la referida al darwinismo como teoría científica de gran significación en las décadas finales del siglo XIX. Se realizó un estudio del contenido de la publicación, con el objetivo de rastrear las referencias al darwinismo y cómo fueron aprovechadas por José Martí en su propósito educativo.

Palabras clave: José Martí, La Edad de Oro, darwinismo.

\section{Abstract}

This report approaches a topic linked to the presence of the science like part of the humanistic formation with which José Marti sought to educate the children from America by means of its magazine The Age of Gold. In this sense a thematic one is assumed that is implicit in several of their texts, the one referred to the Darwinism like scientific theory of great significance in the final decades of the XIX century. He/she was carried out a study of the content of the publication, with the objective of raking the references to the Darwinism and how they were taken advantage of by José Marti in their educational purpose.

Keywords: José Martí, The Age of Gold, darwinism.
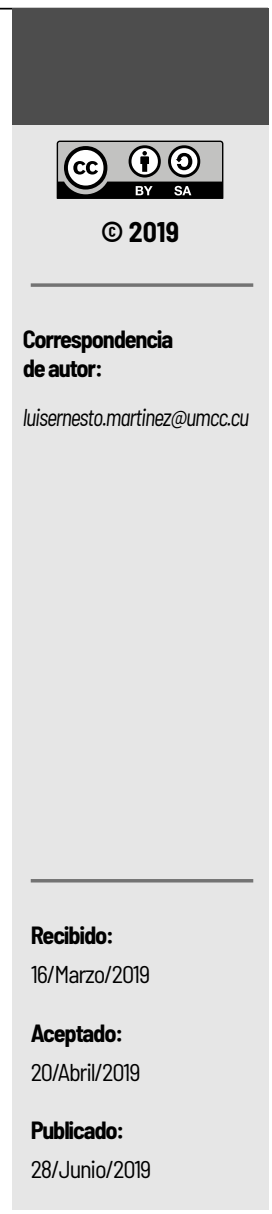


\section{Introducción}

Cuando se publicó La Edad de Oro en 1889, eran evidentes dos cuestiones relativas a la teoría de Darwin sobre la evolución de la vida: el darwinismo había triunfado de forma terminante y definitiva en el pensamiento científico de su tiempo $y$, por otra parte, José Martí había dado amplias muestras de dominar las esencias de esta teoría y valoraba como altamente positivos los aportes de Darwin al estudio de la naturaleza. Para la investigadora Mariana Serra (2009), los principales aspectos relacionados con el darwinismo en La Edad de Oro se reflejan en el artículo "Cuentos de elefantes", cuestión que es válida. No obstante, otros contenidos de la revista reflejan también, de alguna manera, la influencia de los estudios realizados por Martí en torno a Darwin y el darwinismo, como es lo relativo al origen de la especie humana, al poder adquirido por el ser humano al estudiar la naturaleza, así como una referencia implícita a una de las obras más conocidas de Darwin, entre otros elementos que se irán detallando.

\section{Desarrollo}

Precisamente se comenzará por esta referencia, la más clara de cuantas se pueden rastrear en La Edad de Oro. Se trata del cuento "Bebé y el señor Don Pomposo", aparecido en el primer número de la publicación, donde al final del primer párrafo se expresa:

cada vez que ve Bebé a su mamá, le echa el bracito por la cintura, o se le sienta al lado en la banqueta, a que le cuente cómo crecen las flores, y de dónde le viene la luz al sol y de qué está hecha la aguja con que cose, y si es verdad que la seda de su vestido la hacen unos gusanos, y si los gusanos van fabricando la tierra, como dijo ayer en la sala aquel señor de espejuelos. (Martí, 1975b, pp.344-345)

Esta referencia a esos gusanos, en realidad lombrices de tierra, se corresponde con el contenido del libro de Darwin titulado The formation of vegetable mould, through the action of worms, publicado en 1881 y sobre el cual José Martí ya había dado noticias 
en su "Sección constante" del 2 de enero de 1882, publicada en el diario La Opinión Nacional, de Caracas.

En "La historia del hombre contada por sus casas" hay otras resonancias que remiten a Darwin. Comienza Martí un hermoso recuento de la prehistoria humana, sus características y condicionantes ambientales. Recordó que "...grandes eran entonces los animales, grandes como montes..." (Martí, 1975f, p.354). Similar analogía ya había utilizado cinco años atrás, en "Darwin ha muerto", cuando recreó el asombro del inglés ante los monumentales restos de los grandes mamíferos sudamericanos ya extintos:

Y más allá iqué magnífica sorpresa! -Alli están los roedores gigantescos, testigos de otros mundos; restos de megalonix; huesos de megaterio; vestigio del gran caballo americano. $Y$ qué ancas las de esas bestias montañosas! qué garras, que parecen troncos de árbol! Y se sentaban al pie de aquellos árboles colosos, y abrazados a ellos, traían a sí las ramas, con estruendo de monte que se despeña, y comían de ellas! (Martí, 2007, pp.200-202)

Este artículo, donde Martí recrea los aportes de diferentes épocas y regiones al desarrollo de la vivienda como expresión cultural de la civilización humana, asoma en varias oportunidades lo relativo al desarrollo histórico del ser humano, aunque se trate de su evolución social y no biológica, principalmente. No obstante, se observa en todo el texto la influencia de los hallazgos de fósiles humanos en la segunda mitad del siglo XIX, los cuales fundamentaron los criterios darwinistas sobre el origen del hombre $y$, por tanto, estuvieron en el centro de las polémicas en torno al tema.

Debe recordarse que los primeros fósiles del hombre de Neandertal (Homo neanderthalensis), especie extinta del género Homo que habitó Europa y partes de Asia occidental desde hace 230.000 hasta 28.000 años atrás, durante el Pleistoceno y culturalmente considerados en el Paleolítico medio, fueron descubiertos en Engis, Bélgica, en 1829. Le siguieron otros en Gibraltar, en 1848, pero en su momento no fueron reconocidos como significativos. Esto ocurrió a partir de 1856, cuando aparecieron cerca de Düsseldorf en el valle del río Düssel en Alemania, conocido como 
valle de Neander, los restos descubiertos por Johann Karl Fuhlrott y descritos en 1857 por Hermann Schaaffhausen.

Halladas tres años antes de que Darwin publicara The origin of species..., estas evidencias aportaron las primeras referencias científicamente comprobadas acerca del origen del hombre. En la década siguiente, específicamente en 1868, nueve años después de la aparición de su libro máximo y tres antes de The descent of man..., eran descubiertos los restos fósiles del hombre de Cro-Magnon. El autor del hallazgo fue Louis Lartet, quien reconoció cinco esqueletos en la cueva de Cro-Magnon, en Dordogne, Francia, razón por las que fueron denominados así. Reconocidos como Homo sapiens, fueron estudiados por destacados antropólogos como Jean Louis de Quatrefages y Paul Broca.

Las descripciones que realizó Martí en "La historia del hombre contada por sus casas" sobre las características de la llamada Edad de Piedra, se corresponden con estos descubrimientos y las investigaciones que de ellos se derivaron. Por ello, mencionó esta época por su nombre.

(...) cuando los hombres vivían casi desnudos, o vestidos de pieles, peleando con las fieras del bosque, escondidos en las cuevas de la montaña, sin saber que en el mundo había cobre ni hierro allá en los tiempos que llaman «paleolíticos»... (Martí, 1975f, p.354)

Más adelante volvió a insistir en que

La edad de piedra fue al empezar a vivir, que los hombres andaban errantes huyendo de los animales, y vivían hoy acá y mañana allá, y no sabían que eran buenos de comer los frutos de la tierra (Martí, 1975f, p.358).

Detalló Martí los modos en que vivían aquellos primeros representantes de la especie humana:

Entonces los hombres vivían en las cuevas de la montaña, donde las fieras no podían subir, o se abrían un agujero en la tierra, y le tapaban la entrada con una puerta de ramas de árbol; o hacían con ramas un 
techo donde la roca estaba como abierta en dos; o clavaban en el suelo tres palos en pico, y los forraban con las pieles de los animales que cazaban... (Martí, 1975f, p.354)

Estas referencias martianas se corresponden con sus lecturas de las obras del científico inglés John Lubbock, ferviente darwinista, quien propuso el término paleolítico para la Edad de Piedra y describió en sus obras la cultura humana de aquellos tiempos.

Otro fragmento, en este caso sobre las evidencias que iban quedando a lo largo del tiempo sobre la formación de la tierra, tanto en lo natural como en lo cultural del ser humano, principalmente, puede considerarse también deudor de estas lecturas del Apóstol:

La tierra va echando capas conforme van pasando siglos: la tierra es como un pastel de hojaldres, que tiene muchas capas una sobre otra, capas de piedra dura, y a veces viene de adentro, de lo hondo del mundo, una masa de roca que rompe las capas acostadas, y sale al aire libre, y se queda por encima de la tierra, como un gigante regañón, o como una fiera enojada, echando por el cráter humo y fuego: así se hacen los montes y los volcanes. Por esas capas de la tierra es por donde se sabe cómo ha vivido el hombre, porque en cada una hay enterrados huesos de él, y restos de los animales y árboles de aquella edad, y vasos y hachas; y comparando las capas de un lugar con las de otro se ve que los hombres viven en todas partes casi del mismo modo en cada edad de la tierra: sólo que la tierra tarda mucho en pasar de una edad a otra, y en echarse una capa nueva... (Martí, 1975f, pp.360-361)

Más adelante, en el artículo "La Exposición de París", reafirma el criterio de que Martí conocía los últimos hallazgos de restos fósiles humanos en Europa. En este caso se trata de su comentario en relación con las figuras de diferentes razas humanas representadas en la exposición y llama la atención sobre los que "...están sentados por tierra, trabajando el pedernal, como los que desenterraron en Dinamarca hace poco, cabezudos y fuertes, los hombres de la edad de bronce". (Martí, 1975e, p.415). 
Otra referencia martiana expone a sus lectores las evidencias del arte primitivo, en particular las pinturas encontrada en cuevas. Dice Martí:

desde que vino al mundo le gustó al hombre copiar en dibujo las cosas que veía, porque hasta las cavernas más oscuras donde habitaron las familias salvajes están llenas de figuras talladas o pintadas en la roca: y por los montes y las orillas de los ríos se ven manos, y signos raros, y pinturas de animales, que ya estaban allí desde hacía muchos siglos cuando vinieron a vivir en el país los pueblos de ahora. (Martí, 1975e, p.361)

No debió estar ajeno Martí al hallazgo de las pinturas rupestres en Altamira, España, las más célebres de cuantas se conocen. Descubiertas en 1879, aunque la cueva lo había sido desde 1868, fueron el centro de una ardiente polémica entre creacionistas y evolucionistas. Entre los partidarios de dar crédito a la autenticidad de las pinturas, estaba precisamente el paleontólogo español Juan Vilanova, sobresaliente antidarwinista, acerca de cuya obra científica Martí ofreció varios criterios, para quien la calidad de estos dibujos evidenciaba que el hombre no había evolucionado, pues había sido creado por Dios tal y como se le conocía en el siglo XIX. Esto explicaba la perfección y calidad de esas obras artísticas.

Aprovechó esta oportunidad el Apóstol, para resumir algunas de las ideas que defendió en relación con el origen del hombre. La identidad de la especie humana, su diverso origen geográfico y la influencia del medio natural en su desarrollo, son otra vez sustentadas en esta revista para niños, en un lenguaje asequible sin dejar de ser científico y literariamente hermoso. Comentó entonces:

Estudiando se aprende eso: que el hombre es el mismo en todas partes, y aparece y crece de la misma manera, y hace y piensa las mismas cosas, sin más diferencia que la de la tierra en que vive, porque el hombre que nace en tierra de árboles y de flores piensa más en la hermosura y el adorno, y tiene más cosas que decir, que el que nace en una tierra fría, donde ve el cielo oscuro y su cueva en la roca. $Y$ otra cosa se aprende, y es que donde nace el hombre salvaje, sin saber que hay ya pueblos en el mundo, empieza a vivir lo mismo que vivieron los hombres de hace miles de años. (Martí, 1975e, p.357) 
La misma idea se reiteró de forma breve más adelante: "...los hombres aparecieron a la vez, como nacidos de la tierra, en muchos lugares diferentes..." (Martí, 1975e, p.362)

En "Nené traviesa", hay un fragmento que resulta muy llamativo. Cuando la protagonista del cuento está hojeando el libro que su padre le prohibió tocar, de pronto se encuentra con una lámina que representa, según parece, diferentes especies de monos. Dice La Edad de Oro:

Un mundo de monos es la otra pintura. Las dos hojas del libro están llenas de monos: un mono colorado juega con un monito verde: un monazo de barba le muerde la cola a un mono tremendo, que anda como un hombre, con un palo en la mano: un mono negro está jugando en la yerba con otro amarillo... (Martí, 1975i, p.378)

No deja de ser relevante la referencia a este mono que caminaba como un hombre, y a la vez reveladora de que el Apóstol quizás, ya asumía como valedero el principal argumento de los que defendían el origen común de los seres humanos y los actuales monos antropomorfos: el parecido físico y anatómico. Por el adjetivo de "tremendo", que destacaba su tamaño y corpulencia, quizás se trataba de un orangután 0 un gorila.

En el citado trabajo de Mariana Serra (2009), esta autora sustentó su análisis sobre la "Presencia de Darwin en La Edad de Oro", principalmente, en el artículo "Cuentos de elefantes". La elección es acertada, pues Martí narra a sus lectores descubrimientos que, aunque realizados muchos años antes de que Darwin publicara The origin of species..., fueron muy favorables para la aceptación del darwinismo.

Dijo el Apóstol al respecto:

Por Siberia sacan de los hielos colmillos del mamut, que fue el elefante peludo, grande como una loma, que ha estado en la nieve, en pie, cincuenta mil años. Y un inglés, [William Edmond] Logan, dice que no son cincuenta mil, sino que esas capas de hielo se fueron echando sobre la tierra como un millón de años hace, y que desde entonces, desde hace un millón de años, están enterrados en la nieve dura los elefantes peludos". (Martí, 1975c, p.486) 
Llama la atención que Martí, desde este primer fragmento sobre el tema del mamut, Ios llamara "elefantes peludos", aunque se trataba de dos especies diferentes. Lo hace no solo para que puedan entenderse sus explicaciones, sino porque en su tiempo era criterio generalizado que los elefantes se derivaban, evolutivamente, de estos gigantes de la edad de hielo.

Continúa diciendo Martí:

Allí se estuvieron en los hielos duros de Siberia, hasta que un día iba un pescador por la orilla del río Lena, donde de un lado es de arena la orilla, y de otro es de capas de hielo, echadas una encima de otra como las hojas de un pastel, y tan perfectas que parecen cosa de hombre esas leguas de capas. Y el pescador iba cantando un cantar, en su vestido de piel, asombrado de la mucha luz, como si estuviese de fiesta en el aire un sol joven. El aire chispeaba. Se oían estallidos, como en el bosque nuevo cuando se abre una flor. De las lomas corría, brillante y pura, un agua nunca vista. Era que se estaban deshaciendo los hielos. Y allí, delante del pobre Shumarkoff, salían del monte helado los colmillos, gruesos como troncos de árboles, de un animal velludo, enorme, negro. Como vivo estaba, y en el hielo transparente se le veía el cuerpo asombroso. Cinco años tardó el hielo en derretirse alrededor de él, hasta que todo se deshizo, y el elefante cayó rodando a la orilla, con ruido de trueno. Con otros pescadores vino Shumarkoff a llevarse los colmillos, de tres varas de largo. Y los perros hambrientos le comieron la carne, que estaba fresca todavía, y blanda como carne nueva: de noche, en la oscuridad, de cien perros a la vez se oía el roer de los dientes, el gruñido de gusto, el ruido de las lenguas. Veinte hombres a la vez no podían levantar la piel crinuda, en la que era de a vara cada crin. $Y$ nadie ha de decir que no es verdad, porque en el museo de San Petersburgo están todos los huesos, menos uno que se perdió; y un puñado de la lana amarillosa que tenía sobre el cuello. De entonces acá, los pescadores de Siberia han sacado de los hielos como dos mil colmillos de mamut. (Martí, 1975c, pp.486-487) 
Esta historia que Martí recreó de forma magistral fue real. La primera vez que se encontró un ejemplar completo de mamut congelado fue en 1806, en la desembocadura del río Lena, en Siberia. Es conocido como el Mamut de Adams por el apellido del botánico Mikhail Adams, quien supo de su existencia cuando se encontraba cerca de aquella región, identificó sus restos e hizo su descripción científica. Martí no desconoció el curioso detalle, según quedó registrado, de que su cuerpo había sido mordido por lobos en épocas posteriores a su congelamiento, aunque hizo resaltar que su carne había alimentado perros hambrientos.

Terminó entonces, la parte del artículo dedicada al mamut y estableció una emotiva comparación entre esta especie y el elefante africano:

A miles parece que andaban los mamuts, como en pueblos, cuando los hielos se despeñaron sobre la tierra salvaje, hace miles de años; y como en pueblos andan ahora, defendiéndose de los tigres y de los cazadores por los bosques de Asia y de África; pero ya no son velludos, como los de Siberia, sino que apenas tienen pelos por los rincones de su piel blanda y arrugada... (Martí, 1975c, p.487)

Vale aclarar que el mamut lanudo o mamut de la tundra (Mammuthus primigenius), era una especie de mamífero proboscídeo de la familia de los elefántidos. Tenía la piel cubierta de un pelo largo enmarañado, lo cual le permitía a esta especie adaptarse al clima extremo de su época. Se han hallado muchos restos óseos de esta especie, entre los que sobresalen sus grandes colmillos, así como cuerpos enteros congelados, en Siberia, las islas del Ártico y Norteamérica. Están descritas cerca de once especies de mamuts, de diferente tamaño y peso.

No andaba muy alejado Martí de la realidad cuando suponía un parentesco evolutivo entre el mamut y el elefante, criterio muy común en su época. Aunque se basó para ello en el parecido físico, en la actualidad la genética confirma esta apreciación. De acuerdo con las investigaciones más recientes, la divergencia del mamut lanudo, los elefantes africanos y el elefante asiático ocurrió en un breve período, y confirma que el mamut estaba más relacionado con la especie asiática que con la africana. En el 2005 un equipo de investigadores estadounidenses, alemanes y británicos fueron capaces de reunir un perfil de ADN mitocondrial completo del mamut, lo cual permitió delinear la cercana relación evolutiva entre estos y los elefantes asiáticos. 
Los elefantes africanos se separaron del mamut lanudo hace cerca de 6 millones de años, más o menos por la misma época en que se produjo la separación evolutiva entre los chimpancés y los humanos.

Vale advertir que en el Journal of researches..., libro que Martí estudió profundamente, Darwin reflexionó que

En Siberia también se encuentran bosques de abedules, abetos, álamos y alerces, a una latitud ( 64 grados) en que la temperatura media del aire está bajo cero y la tierra helada tan completamente, que el cadáver de un animal enterrado en ella se conserva de un modo perfecto. (Darwin, 1978, p.158)

Para después hacer alusión a "...los animales que se han encontrado en Siberia conservados en el hielo" (Darwin, 1978, p.158). En un tercer momento volvió sobre el tema, pero esta vez mencionando concretamente los ejemplares encontrados en esa remota región. Nótese que no utiliza el término mamut, sino elefante: "La perfecta conservación de los cadáveres de los elefantes y rinocerontes de Siberia es con seguridad uno de los fenómenos más extraños de la geología..." (Darwin, 1978, p.425)

Otro fragmento de este artículo que se relaciona con los conocimientos de Martí acerca de Darwin y el darwinismo, es su descripción de la trompa del elefante. Puede considerarse así, porque es magistral la forma en que revela la relación estructura-función de esta importante forma anatómica. Para el desarrollo de la Biología como ciencia, la comprensión del desarrollo de esta relación en correspondencia con las condiciones ambientales, fue un factor esencial que tuvieron en cuenta las teorías evolucionistas de Lamarck y Darwin. Sobre el tema expuso el Apóstol:

La trompa es lo que más cuida de todo su cuerpo recio el elefante, porque con ella come y bebe, y acaricia y respira, y se quita de encima los animales que le estorban, y se baña. Cuando nada iy muy bien que nadan los elefantes! no se le ve el cuerpo, porque está en el agua todo, sino la punta de la trompa, con los dos agujeros en que acaban las dos canales que atraviesan la trompa a lo largo, y llegan por arriba a la misma nariz, que tiene como dos tapaderas, que abre y cierra según 
quiera recibir el aire, o cerrarle el camino a lo que en las canales pueda estar. Nadie diga que no es verdad, porque hay quien se ha puesto a contarlos: como cuarenta mil músculos tiene la trompa del elefante, la «proboscis», como dice la gente de libros: toda es de músculos, entretejidos como una red: unos están a la larga, de la nariz a la punta, y son para mover la trompa adonde el elefante quiere, y encogerla, enroscarla, subirla, bajarla, tenderla: otros son a lo ancho, y van de las canales a la piel, como los rayos de una rueda van del eje a la llanta: esos son para apretar las canales o ensancharlas. ¿Qué no hace el elefante con su trompa? La yerba más fina la arranca del suelo. De la mano de un niño recoge un cacahuete. Se llena la trompa de agua, y la echa sobre la parte de su cuerpo en que siente calor. Los elefantes enseñados se quitan y se ponen la carga con la trompa. Un hilo levantan del suelo, y como un hilo levantan a un hombre. (Martí, 1975c, pp.487-488)

La trompa es lo más notable de la anatomía del elefante y se originó como resultado de la transformación del labio superior y de la nariz en un órgano alargado, muscular y carente de huesos. Este apéndice es utilizado para arrancar hierbas y hojas para alimentarse o para succionar agua. Con la trompa, que en el elefante africano termina en dos prolongaciones digitiformes y en el asiático en solo una, es capaz de coger con precisión y hasta con delicadeza, cualquier objeto pequeño, como puede ser una hoja. Es un órgano de gran sensibilidad, capaz de percibir olores y sabores. Con ella también pueden emitir sonidos, derribar árboles, desgarrar vegetación 0 darse baños de polvo y agua. Fue el biólogo francés Georges Cuvier, famoso por su férrea oposición a la evolución de las especies, quien consideró en 40000 el número de músculos de la trompa del elefante. Su estudio de este singular órgano formó parte de los que reunió en la obra Leçons d'anatomie comparée (1800-1805), la cual publicó en cinco volúmenes.

Otro elemento que permite establecer una relación entre lo que escribió Martí para La Edad de Oro y sus criterios sobre Darwin, es la defensa constante de la necesidad de aprender, su reconocimiento de la bondad como virtud íntimamente relacionada con el saber, así como el gusto que debía sentir cada ser humano al estudiar la naturaleza. Darwin fue para Martí uno de aquellos hombres buenos que mencionó en La Edad de Oro, de los que había "...dicho algo útil a los demás" (Martí, 1975h, p.455). 
No en balde en el ensayo a propósito de su muerte en 1882, lo retrató como alguien en quien "...resplandecía el orgullo de haber visto" (Martí, 2007, p.180), de mirada "... benévola, cual la de aquellos que viven en trato fecundo con la naturaleza, y su mano blanda y afectuosa, como hecha a cuidar pájaros y plantas..."(Martí, 2007, pp.180-181).

El amor por el trabajo fue otra virtud que resaltó en Darwin, a quien imaginó "... memorando laboriosamente..." (Martí, 2007, p.182), en su casa de Down House "...en su hermosísimo cuarto de estudiar, repleto de huesos y de flores, y de cierta luz benigna que tienen los cuartos en que se piensa honestamente..."(Martí, 2007, p.182).

En La Edad de Oro recordó como a santos a "...los hombres que desean saber...", pues "...los hombres deben aprenderlo todo por sí mismos, y no creer sin preguntar, ni hablar sin entender, ni pensar como esclavos lo que les mandan pensar otros..." (Martí, 1975k, p.459).

Como una invitación martiana a estudiar las ideas de todos los pensadores revolucionarios de la humanidad, entre ellos Darwin, queda este hermoso párrafo que dedicó a los niños de América:

lo que se ha de hacer es estudiar con cariño lo que los hombres han pensado y hecho, y eso da un gusto grande, que es ver que todos los hombres tienen las mismas penas, y la historia igual, y el mismo amor, y que el mundo es un templo hermoso, donde caben en paz los hombres todos de la tierra, porque todos han querido conocer la verdad, y han escrito en sus libros que es útil ser bueno, y han padecido y peleado por ser libres, libres en su tierra, libres en el pensamiento. (Martí, 1975k, p.460)

La necesidad de indagar de forma sistemática para entender el mundo, en lo cual Darwin era un ejemplo de perseverancia, la retomó cuando señaló: "Y no es que uno no quiere saber; porque la verdad es que da vergüenza ver algo y no entenderlo, y el hombre no ha de descansar hasta que no entienda todo lo que ve"(Martí, 1975d, p.471). Eso tenía como recompensa la felicidad plena: "Cuando uno sabe para lo que sirve todo lo que da la tierra, y sabe lo que han hecho los hombres en el mundo, siente uno deseos de hacer más que ellos todavía: y eso es la vida" (Martí, 1975d, p.471). 
La frase con que Martí cerró el cuarto número de La Edad de Oro, última de toda la revista, plantea: "Hay que ir de vez en cuando a vivir en lo natural, y a conocer la selva" (Martí, 1975h, p.503). Esta idea, realmente es una constante en la cultura científica martiana, se relaciona con varios pasajes que expresara en "Darwin ha muerto" al seguir la lectura del Journal of researches..., específicamente con aquellos que tratan sobre las vivencias de Darwin al estudiar la selva brasileña.

\section{Conclusiones}

Darwin y el darwinismo estuvieron presentes en el proyecto martiano concretado en La Edad de Oro. Además de lo ya planteado, se debe tener en cuenta lo que escribió en el prospecto de la revista. Uno de sus propósitos era precisamente poner a sus lectores en contacto con la poesía "...sana y útil que nace del conocimiento del mundo..." (Martí, 1975j, p.296), para lo cual se estudiarían preferentemente "...las leyes, agentes e historia de la tierra donde ha de trabajar por la gloria de su nombre y las necesidades del sustento" (Martí, 1975j, p.296). Para lograrlo era necesario que: "Los temas escogidos serán siempre tales que, por mucha doctrina que lleven en sí, no parezca que la llevan, ni alarmen al lector de pocos años con el título científico ni con el lenguaje aparatoso" (Martí, 1975j, p.296).

Como máxima figura científica de su época, Darwin era un ejemplo que debían conocer los niños de su tiempo, así como también dominar los aspectos básicos relacionados con el darwinismo como teoría científica. Esto hizo Martí en La Edad de Oro, aún cuando no mencionara al científico inglés por su nombre ni incluyera en sus páginas un resumen de su teoría. Los ejemplos incluidos en este análisis demuestran la maestría martiana para enseñar estos temas "como sin querer" (Martí, 1975g, p.330) y el cumplimiento de su promesa expuesta en "A los niños que lean La Edad de Oro": "Les vamos a decir cómo está hecho el mundo: les vamos a contar todo lo que han hecho los hombres hasta ahora."(...) "...les diremos lo que se sabe del cielo, y de lo hondo del mar y de la tierra..." (Martí, 1975a, pp.301 y 302).

\section{Referencias Bibliográficas}

Darwin, C. (1978). Viaje de un naturalista alrededor del mundo. La Habana, Cuba: Gente Nueva. 
Martí, J. (1975a). A los niños que lean la edad de oro, obras completas (tomo 18). La Habana, Cuba: De Ciencias Sociales.

Martí, J.(1975b). Bebé y el señor Don Pomposo, obras completas (tomo 18). La Habana, Cuba: De Ciencias Sociales.

Martí, J. (1975c). Cuentos de Elefantes, obras completas (tomo 18). La Habana, Cuba: De Ciencias Sociales.

Martí, J. (1975d). Historia de la cuchara y el tenedor, obras completas (tomo 18). La Habana, Cuba: De Ciencias Sociales.

Martí, J. (1975e). La exposición de París, obras completas (tomo 18). La Habana, Cuba: De Ciencias Sociales.

Martí, J. (1975f). La historia del hombre contada por sus casas, obras completas (tomo 18). La Habana, Cuba: De Ciencias Sociales.

Martí, J. (1975g). La llíada de Homero, obras completas (tomo 18). La Habana, Cuba: De Ciencias Sociales.

Martí, J. (1975h). La última página, obras completas (tomo 18). La Habana, Cuba: De Ciencias Sociales.

Martí, J. (1975i). Nené traviesa, obras completas (tomo 18). La Habana, Cuba: De Ciencias Sociales.

Martí, J. (1975j). Nota preliminar, obras completas (tomo 18). La Habana, Cuba: De Ciencias Sociales.

Martí, J. (1975k). Un paseo por la tierra de los Anamitas, obras completas (tomo 18). La Habana, Cuba: De Ciencias Sociales.

Martí, J. (2007). Darwin ha muerto, obras completas, (tomo 11, ed. crítica). La Habana, Cuba: Centro de Estudios Martianos.

Serra, M. (2009). Presencia de Darwin en la edad de oro. Imaginarios: La edad de oro (1889). Recuperado de: http://librinsula.bnjm.cu/secciones/247/ expedientes/247_exped_1.html 